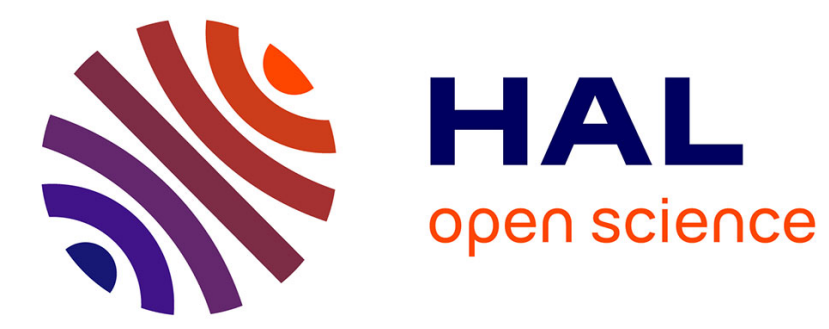

\title{
Aeroacoustic investigation of a single airfoil tip leakage flow
}

\author{
Marc C. Jacob, Julien Grilliat, R. Camussi, G.C. Gennaro
}

\section{To cite this version:}

Marc C. Jacob, Julien Grilliat, R. Camussi, G.C. Gennaro. Aeroacoustic investigation of a single airfoil tip leakage flow. International Journal of Aeroacoustics, 2010, 9 (3), pp.253-272. 10.1260/1475472X.9.3.253 . hal-00566045

\section{HAL Id: hal-00566045 https://hal.science/hal-00566045}

Submitted on 8 Jun 2012

HAL is a multi-disciplinary open access archive for the deposit and dissemination of scientific research documents, whether they are published or not. The documents may come from teaching and research institutions in France or abroad, or from public or private research centers.
L'archive ouverte pluridisciplinaire HAL, est destinée au dépôt et à la diffusion de documents scientifiques de niveau recherche, publiés ou non, émanant des établissements d'enseignement et de recherche français ou étrangers, des laboratoires publics ou privés. 


\title{
Aeroacoustic investigation of a single airfoil tip leakage flow
}

\author{
M. C. Jacob1, J. Grilliat ${ }^{1}$, R. Camussi ${ }^{2}$ and G. Caputi Gennaro² \\ ${ }^{1}$ Centre Acoustique du LMFA, UMR CNRS 5509 - Ecole Centrale de Lyon - Université \\ Claude-Bernard Lyon I F-69134 Ecully Cedex \\ 2Mechanical and Industrial Engineering Dept. (DIMI) \\ University 'Roma 3', I-00146, Rome \\ marc.jacob@ec-lyon.fr, julien.grilliat@ec-lyon.fr,camussi@uniroma3.it, gcaputi@uniroma3.it
}

Received August 31, 2009; Revised January 18, 2010; Accepted February 2, 2010

\begin{abstract}
Combined near and far field aeroacoustic measurements have been carried out during an original laboratory scale low Mach number (0-0.3) experiment about the tip leakage flow past a single non-rotating airfoil. Such measurements were made possible by the use of a single airfoil located in the potential core of an open jet flow in the anechoic wind tunnel of the Ecole Centrale de Lyon. The airfoil was mounted between two flat plates. A strong tip clearance flow was achieved without rotation by paying a special attention to the choice of the airfoil which was a 5\% camber, $10 \%$ thickness NACA5510 airfoil that provided a high lift at a $15^{\circ}$ angle of attack. The experiment gave rise to an extensive data set obtained with several flow velocity measurement techniques (HWA, LDA, PIV), steady and unsteady pressure measurements on the airfoil and the casing plate as well as far field pressure measurements. Further, cross-analyses of various velocity and pressure signals allowed to locating sources and identifying their mechanisms. Results showed evidence of two components of tip leakage broadband self noise.
\end{abstract}

\section{INTRODUCTION}

Fan broadband noise appears to be a major sound source of modern aircraft during approach flights where the engine power and the jet noise are reduced. There are many types of broadband noise sources in fan-OGV secondary flows, not to mention compressor, turbine, and combustion noise that are believed to be either small or immersed in the jet noise. These sources can be decomposed in two families, self noise and interaction noise sources.

Self noise sources are due to flow perturbations generated by an airfoil that are converted into sound by the same airfoil. They are mainly located on the rotor and are known as trailing edge noise and tip clearance noise sources. Perturbations generated at the hub are aerodynamically important but not acoustically since the azimuthal velocity is much lower at the hub; stator self noise exists in principle but is outranged by interaction noise. 
Interaction noise sources are due to perturbations advected by the flow onto a blade that are converted into sound when they hit the blade leading edge. Although these perturbations can be of any nature ( $e g$. ingested atmospheric turbulence), they usually originate from another airfoil wake: the main interaction noise source in the secondary flow is the fan-OGV interaction, due to the rotor wakes impinging onto the stator vanes. Since the rotor wakes contain trailing edge perturbations as well as tip clearance perturbations, the interaction noise can also be split accordingly. Another possible interaction noise source is suspected on the rotor tip, the tip-boundary layer interaction that is due to the interaction between the casing boundary layer structures and the fan tip.

Although tip clearance flows have been investigated by many authors, a number of studies [1]-[4] are devoted to axial flow compressors and focus on their aerodynamic performances. More recent cascade flow studies [5]-[7], including recent numerical investigations [8] provide useful information about the unsteady flow features. More specifically, Bindon [1] and Storer and Cumpsty [3], showed that the tip leakage flow consists in a quasi cross-stream jet-like flow from the pressure side to the suction side, that rolls up into a Tip Leakage Vortex (TLV) when interacting with the external flow at the suction side. Experimental studies conducted on a compressor cascade by Muthanna [5], Tang [6] and Intaratep [7], showed that the circulation of the TLV increases as it is washed downstream along the suction side edge, until the vortex detaches from the edge and starts moving away from the suction side towards the pressure side of the next blade. After the vortex detaches, its circulation stops increasing and the vortex slowly disappears when interacting with the blade wakes. As the gap size increases, the position of the vortex detachment moves downstream and the circulation of the vortex increases.

Moreover, a few studies are concerned with the noise due to axial fan or compressor tip flows. Among these, the early and recent work of Fukano et al [9][10], as well as the study of Ganz et al. [11] provide answers as to the importance of tip clearance noise. The latter study does not show tip clearance noise to be a significant noise source of the secondary flow. Kameier and Neise [12][13] among others, carried out a detailed experimental study on a low Mach number axial fan about tip clearance noise and the particular role of rotating instabilities. Since they are carried out on representative rigs, these works do not fully relate noise measurements to specific flow perturbations and illustrate how difficult it is to identify the role of tip clearance noise among other noise sources on a representative fan rig. Nevertheless, among the most recent papers, some even describe attempts to controlling tip clearance noise [13]-[17] by both active and passive devices such as novel tip design, inferring that tip clearance noise is indeed, an important fan noise source.

In order to address the questions which arise about the importance of tip clearance noise and to shed a new light onto the flow mechanisms involved in it, the present study investigates the tip clearance flow on a single non rotating airfoil both from an aerodynamic and an acoustic standpoint. Thus it is focused on tip clearance self noise and compares it to trailing edge noise. By limiting the study to a single airfoil, the tip clearance flow interaction with an airfoil located downstream is automatically excluded. Moreover, rotating instabilities which have been shown to influence the 
sound production of tip clearance flows by Kameier and Neise[12][13], especially for very small gaps, are not taken into account since the airfoil is non rotating and isolated. The choice of a single non rotating airfoil not only allows to studying separately the sound generation mechanisms related to the tip vortex but also to carrying out the experiment in an open jet in order to obtain a far field in a medium at rest using a similar set-up as in ref [18]. A significant tip flow is obtained by loading the airfoil which is substantially cambered. Although the range of the tip clearance gaps and Mach numbers considered hereafter do not match those of a real engine fan, the underlying physical mechanisms of the tip vortex formation in the gap region and its sound radiation are similar.

The overall objectives of the experiment that is briefly reported in the present paper are manifold:

- to classify the tip clearance flow in the hierarchy of fan broadband noise sources,

- to describe its mechanisms with conventional and less conventional tools (in refs. [19] and [20], a wavelet based study based on concepts detailed in [21] is presented),

- to provide a database both for modelling and numerical issues.

The experimental set-up is described in the next section, the mean flow in section 3 and typical unsteady flow features in section 4 . The sound far field and its relation to the flow field are discussed in section 5. Finally, conclusions are summarized in the last section.

\section{DESCRIPTION OF THE EXPERIMENT}

\subsection{Set-up}

The experiment is carried out in the anechoic room $(10 \mathrm{~m} \times 8 \mathrm{~m} \times 8 \mathrm{~m})$ of the Laboratoire de Mécanique des Fluides et d'Acoustique (LMFA), a joint CNRS-ECLUCBLyon-I laboratory located at the Ecole Centrale de Lyon. Air is supplied by a high speed subsonic anechoic wind tunnel at Mach numbers ranging up to 0.3. The set-up is shown in Figure 1: before reaching the duct exit, the air is accelerated by a convergent nozzle from a $560 \mathrm{~mm} \times 560 \mathrm{~mm}$ cross-section to the $450 \mathrm{~mm}$ wide and 200 high test section, where the flow develops into a semi open jet between two horizontal plates.

A NACA5510 profile (5\% camber, $10 \%$ thickness) with a $c=200 \mathrm{~mm}$ chord and variable span $\ell$ is placed into the core of this jet. The airfoil is mounted onto a wooden disk, which allows to tuning the angle of attack. The tip clearance (or gap) between the airfoil and the lower plate is also adjustable, the total height $L(=h+\ell)$ remaining equal to $L=200 \mathrm{~mm}$. A glass window is mounted into the lower plate allowing for PIV and LDA measurements in the vicinity of the airfoil. Passive boundary layer suction is applied to the nozzle exit upstream of the two plates.

\subsection{Flow conditions}

The reference velocity at the exit of the wind tunnel is $U_{0}=70 \mathrm{~m} / \mathrm{s}$, and the turbulence level $u^{\prime} / U_{0}$ is about $0.7 \%$. The corresponding chord-based Reynolds number is $\operatorname{Re}_{c} \sim 930,000$ whereas the Mach number is $M \sim 0.2$. For these inflow parameters, the 


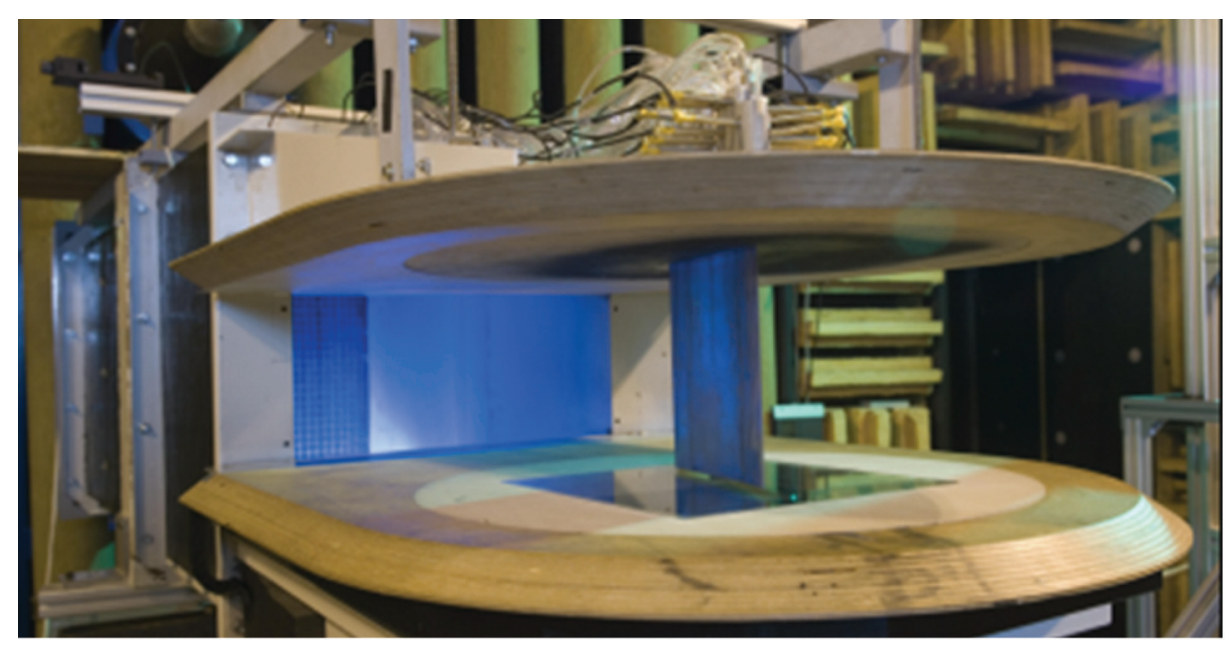

By Courtesy (C) CNRS 2006

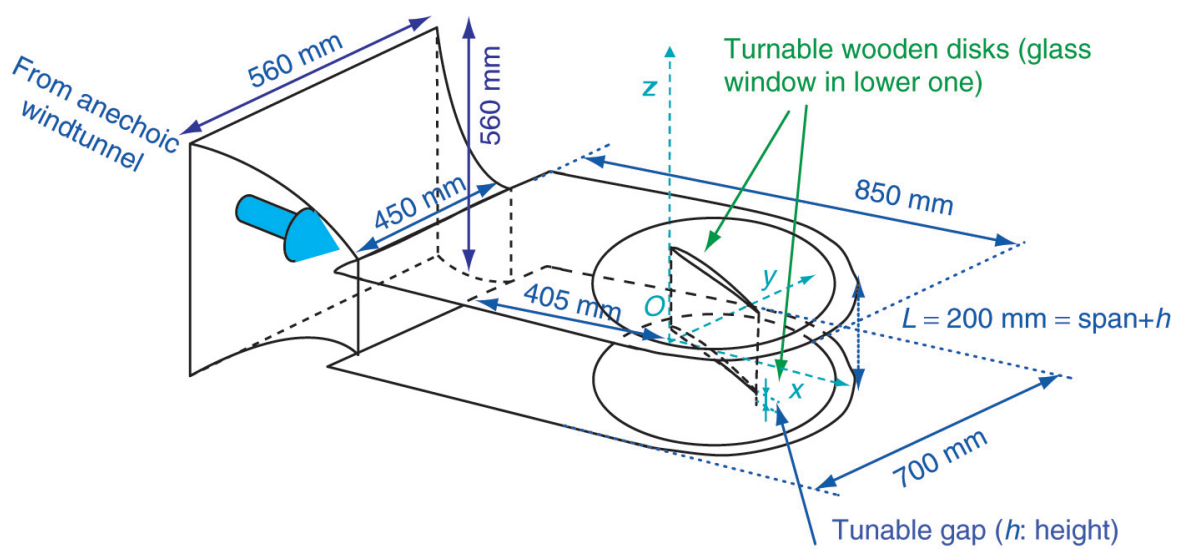

Figure 1: Experimental set-up. Overall picture of the pressure side showing the nozzle exit (blue), the plates with a glass window on the bottom one, the remote pressure probes (Top); sketch of the set-up with co-ordinates and rig dimensions (Bottom).

reference configuration is obtained with a $\alpha=15^{\circ}$ angle of attack, and an $h=10 \mathrm{~mm}$ gap. The corresponding configuration without gap $(h=0)$ is referred to as the no-gap configuration. The gap is half the maximum airfoil thickness $(e=20 \mathrm{~mm})$ and about half the boundary layer thickness (as discussed in section 3). Since the two end plates and the airfoil remain motionless, the gap flow is only induced by the high camber (5\%) and angle of attack $\left(15^{\circ}\right)$. This results in a high load and a subsequently significant gap flow.

Starting from this reference configuration, the inflow velocity, the angle of attack and the tip clearance are varied between $U_{0}=20$ and $90 \mathrm{~m} / \mathrm{s}, \alpha=0$ and $18^{\circ}, h=0$ and $25 \mathrm{~mm}$ respectively. The no-gap configuration gives a comparison point for the non-zero 
gap configurations. Most results shown hereafter are obtained in the reference configuration.

\subsection{Co-ordinate system}

The coordinate system is bound to the airfoil which is useful for describing the wall pressure and the velocity nearby the airfoil. The origin $O$ is located at the leading edge tip: the $x$ axis follows the aerodynamic chord pointing from the leading to the trailing edge; the $z$ axis follows the span-wise direction from the gap to the upper plate and the $y$ axis is normal to the chord, pointing from the pressure to the suction side.

\subsection{Measurements}

Measurements referred to in this paper and described hereafter include HWA, PIV, LDA, wall pressure and far field pressure measurements.

- Both single and cross-wire anemometry using a Dantec anemometer with Dantec wires are carried out to characterize the incoming flow, the flow around the airfoil and the wake. Signals are sampled at $10 \mathrm{kHz}$ for the mean flow statistics and at $45 \mathrm{kHz}$ for the velocity spectra.

- PIV measurements are performed in the vicinity of the airfoil at various spanwise positions $z$, including the tip clearance gap, with a LaVision system and two fast high resolution CCD cameras $(1280 \times 1024$ pixels each $)$ with $35 \mathrm{~mm}$ lenses and interferometric filters located below the glass window. They are located next to each other in order to provide pictures from a $250 \times 105 \mathrm{~mm}^{2}$ rectangular area surrounding the whole airfoil with a good resolution. In order to perform measurements at any span-wise position without tuning the system, both the Laser and the cameras are fitted on a common support that can be moved up and down as sketched on Figure 2 (a). Their axes are normal to the light sheet. Images are post-processed with the LaVision software DaVis. The cameras are placed beneath the glass window whereas the Laser-sheet is directed horizontally first towards the suction side and then towards the pressure side in order to remove shadow zones when $z \geq 0$. The velocity fields are computed using a $32 \times 32$ pixel interrogation window that corresponds to a $3.1 \times 3.2 \mathrm{~mm}^{2}$ area in the image. The resolution is fine enough to avoid significant peak-locking effects and the interrogation zone is both large enough to ensure a good correlation between the camera images and small enough to obtain a well resolved velocity field. The delay between 2 images is about $5-15 \mu \mathrm{s}$ in the gap region and even up to $40 \mu \mathrm{s}$ away from the gap. The flow is seeded with heated paraffin (vapour) injected upstream of the wind tunnel. The time resolution of the method is limited by the characteristics of both the Laser system and the camera. In the present case it is of the order of $4 \mathrm{~Hz}$.

- LDA measurements that are carried out in the gap region use a Dantec dual-beam, backscatter Laser Doppler Anemometer (LDA) system. A photograph is shown on Figure 2 (b). Two pairs of beams are used for two-dimensional velocity measurements. They are supplied by the green line $(514.5 \mathrm{~nm})$ and the blue line $(488 \mathrm{~nm})$ of a Spectra Physics $4 \mathrm{~W}$ argon-ion Laser source. The beams of each pair 


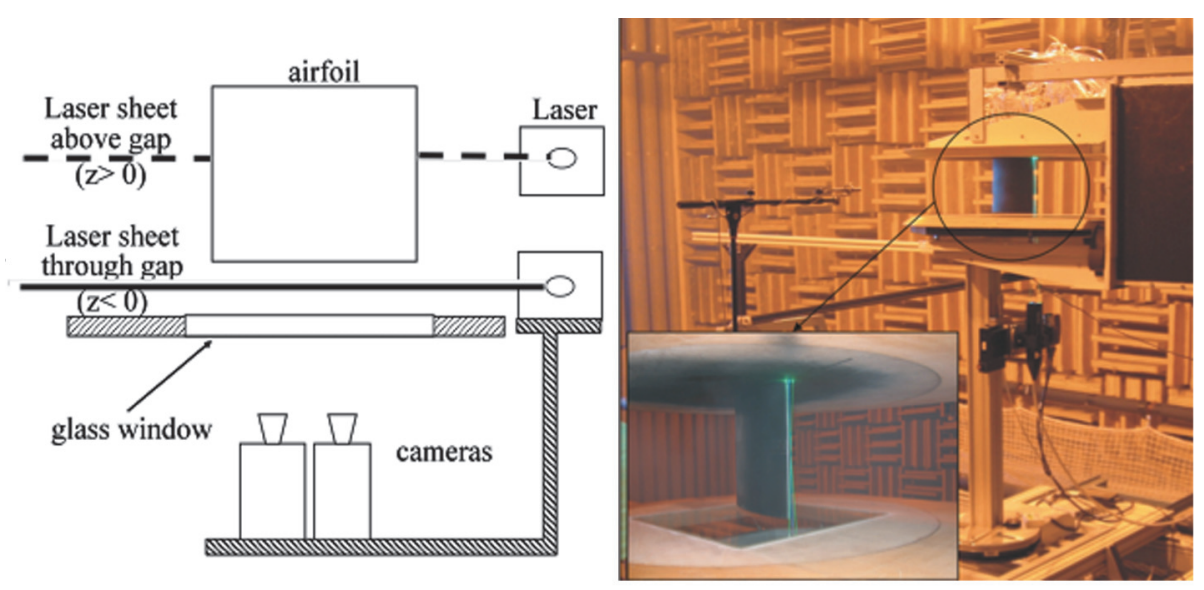

(b) LDA (here at mid-span during calibration)

Figure 2: Set-up for velocity measurements.

undergo a relative frequency shift of $40 \mathrm{MHz}$ in a Bragg cell. The four beams are guided to the flow with an optical fiber which is terminated by a focusing lens with a focal length of $400 \mathrm{~mm}$. The beams of each pair have a mutual angle of $9^{\circ}$. For a measurement of the stream-wise and cross-stream velocity components, each Laser beam makes a $4.5^{\circ}$ angle with the span-wise direction in the plane of the corresponding velocity component. The size of the measurement volume (i.e. the spatial resolution) is about $70-75 \mu \mathrm{m}$ whereas its length in the span-wise direction is $0.9 \mathrm{~mm}$. The fringe spacing is about $3 \mu \mathrm{m}$. The backscattered beams are focused by the same lens and sent through an optical fiber onto photomultipliers. The signals are then treated by two Dantec real-time signal analyzer and post-processed based on $0^{\text {th }}$ order re-sampling. The seeding material is the same as that used for the PIV measurements.

- Both steady and unsteady pressure measurements are carried out on the airfoil and the lower plate, including the gap: the sampling rate is $64 \mathrm{kHz}$ and the time series are long enough to perform 500 averages of 8192 point FFT's; this is enough to obtain a statistical error about $7 \%$ on the coherence between 2 signals. The measurements are carried out with a remote microphone technique described by Roger and Perennes [22]. The sensors are B\&K type 4935 ICP $1 / 4$ " microphones that are pre-amplified by a PXI system; they are connected to the wall measurement pinholes via small tubes. This remote microphone technique requires an appropriate calibration (described in [23]) that takes into account the transfer function of the capillary tubes. Results are relevant up to frequencies reaching $8 \mathrm{kHz}$. The unsteady pressure is measured at 35 locations. The main probes and their locations are shown on Figure 3. The same tubes and a few others are connected to a Furness manometer via a Scanivalve system that allows the remotely controlled acquisition of 48 mean pressure signals. 


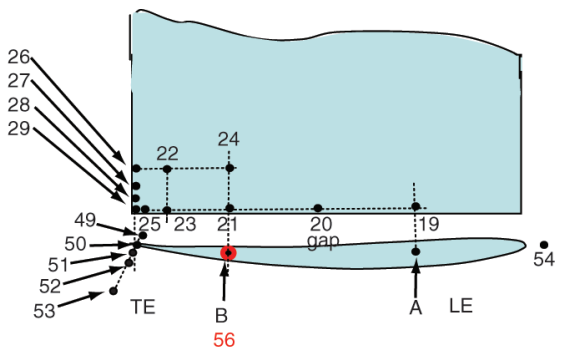

(a) Pressure probe locations in the gap and near the gap on the suction side. Pressure side probes and mid-span probes are not shown here.

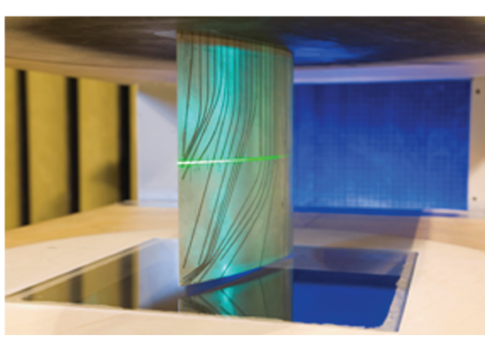

(b) Picture of the airfoil showing TE corner and (near) mid-span probes. The lines correspond to the imbedded tubes.

Figure 3: Sketch and photograph of main pressure probes.

- Far field measurements are performed at $1.7 \mathrm{~m}$ from the airfoil leading edge with two B\&K type 4191 1/2" microphones and B\&K type 2669 preamplifiers placed at each side of the airfoil. The microphones are turned around the airfoil at mid-span in far field conditions above $\sim 250 \mathrm{~Hz}$. For practical reasons the device rotates around a point located at $x=-0.075 c=-15 \mathrm{~mm}$ and $y=0$ from the $(O, z)$ axis. The observation angle is expressed with respect to the airfoil chord and varies within the range $50^{\circ}$ to $160^{\circ}$ across the suction side and from $-130^{\circ}$ to $-20^{\circ}$ across the pressure side.

Some of the techniques described above are applied simultaneously in order to combine various signals such as velocity and pressure signals.

A selection of most remarkable results from this experiment that provided a large data set is described in the present paper.

\section{THE STEADY FLOW}

\subsection{Upstream flow}

Two stream-wise velocity profiles $U / U_{0}$ and the corresponding turbulence levels $u^{\prime} / U_{0}$ are measured with HWA about half a chord $(x / c=-0.52)$ upstream of the airfoil, one in the cross-stream direction at $z=L / 2+h=110 \mathrm{~mm}$ shown on Figure 4(a), the other in the span-wise direction at $y / c=-0.08$ shown on Figure 4(b). The former (see Figure 4 (a)) shows that the potential core flow is uniform within $\pm 3 \%$ in the cross-stream direction and has a very low turbulence level (about $\sim 0.7 \%$ ). The plot also shows the inner parts of the 2 jet shear layers at $y / c \sim+0.5$ and -1 . Figure $4(\mathrm{~b})$ provides information about the boundary layer upstream of the gap: its thickness is $\delta \sim 18 \mathrm{~mm}$, that is, almost twice the gap height in the reference configuration $(h=10 \mathrm{~mm})$ and 18 times the smallest gap $(h=1 \mathrm{~mm})$. The associated displacement thickness is $\delta^{*}=1.4 \mathrm{~mm}$. In the boundary layer, the turbulence level increases up to $8 \%$. Similar conclusions can be drawn from other HWA measurements at the jet nozzle. 

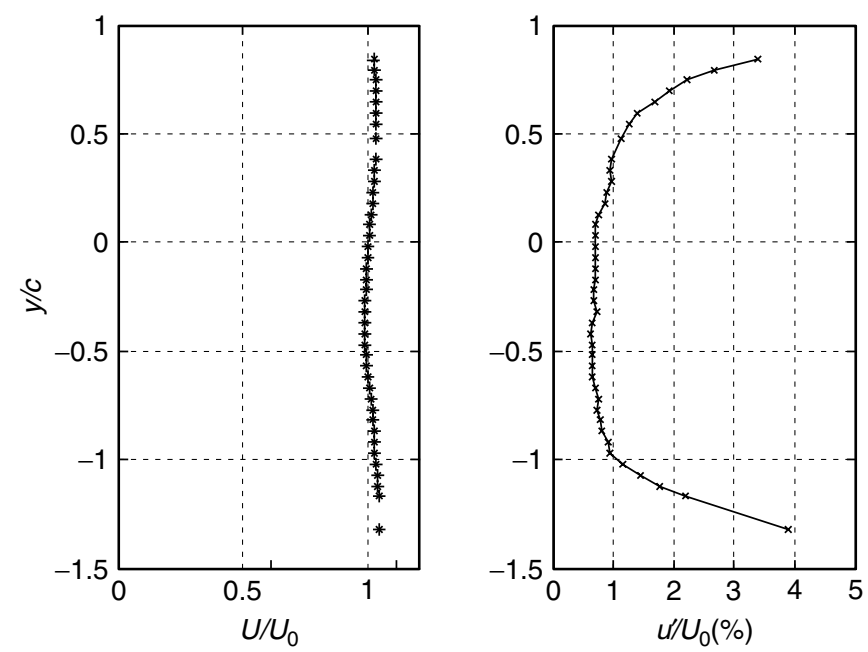

(a) Cross-stream profile at $x / c=-0.52 ; z=L / 2+h$
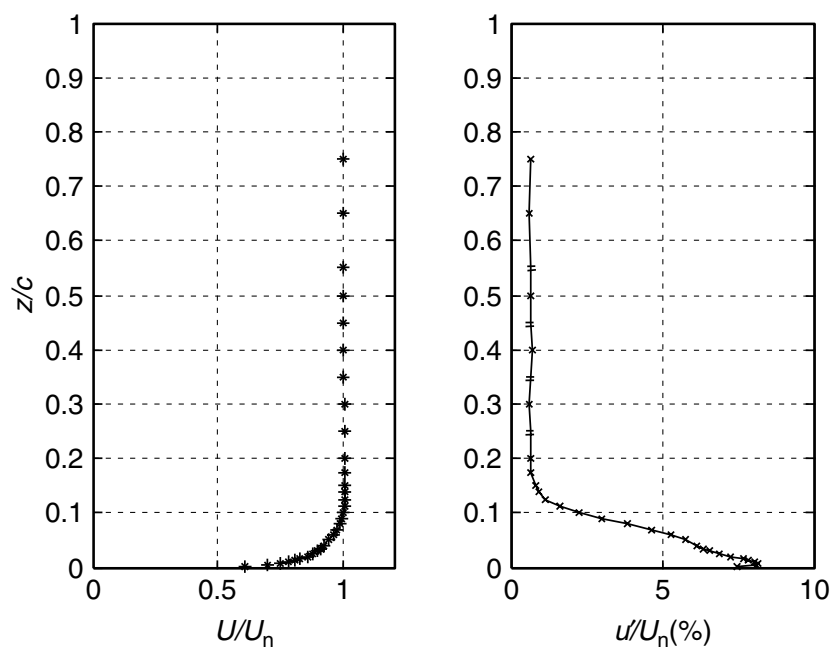

(b) Bottom plate boundary layer profile.

Span-wise profile at $x / c=-0.52 ; y / c=-0.08$

Figure 4: Upstream flow at $70 \mathrm{~m} / \mathrm{s}, h=10 \mathrm{~mm}$ and $\alpha=15^{\circ}$.

\subsection{Pressure coefficient}

In the mid-span region the flow is not significantly affected by the tip clearance flow as shown on Figure 5(a), where the pressure coefficient along the mid-span chord is plotted for various gap heights from $h=0$ to $10 \mathrm{~mm}$. This is confirmed by velocity measurements in the same region. It can be seen that the airfoil is highly loaded but not 


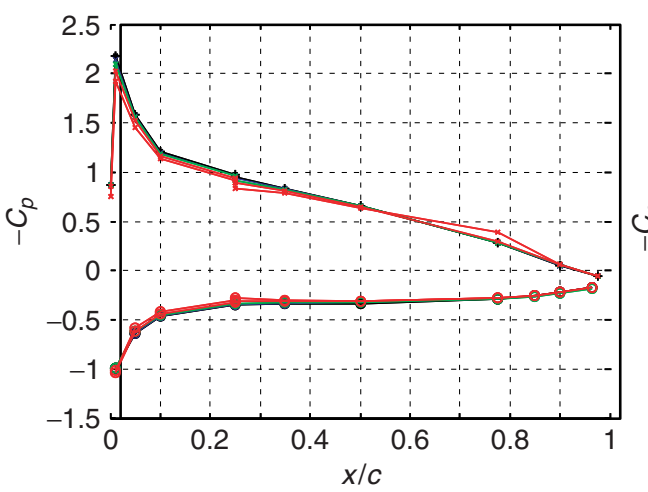

(a) Gap influence onto mid-span pressure at $70 \mathrm{~m} / \mathrm{s}$ : no gap (black); $h[\mathrm{~mm}]=1$ (blue); 2 (green); 5 (red dash); 10 (red continuous)

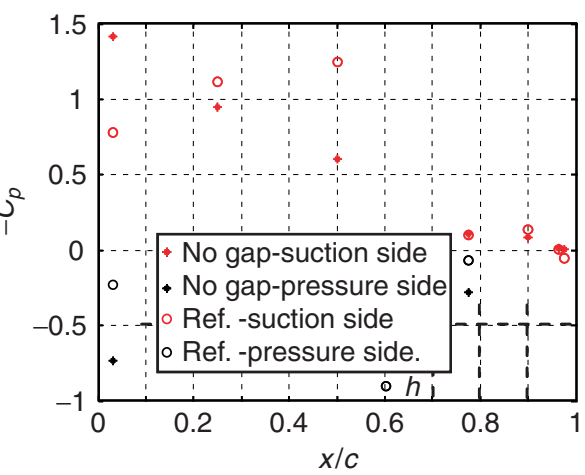

(b) Pressure coefficient at the tip edge: with gap (circles) and without gap (stars)suction side (red) and pressure side (black)

Figure 5: $\quad$ Pressure coefficient at $U_{0}=70 \mathrm{~m} / \mathrm{s}, h=10 \mathrm{~mm} ; \alpha=15^{\circ}$.

stalled. Thus a strong gap flow is expected but the flow separation at the tip does not affect the whole span of the airfoil. As a result, the flow is completely modified in the tip clearance region, as illustrated on Figure 5(b) where the chord-wise pressure coefficient obtained at the tip edge is plotted both with and without gap. Besides the fact that pressure distributions of the two configurations are quite different in this region, it can be observed that for the reference configuration, the suction at mid-chord is significantly increased with respect to the no-gap case.

\subsection{Mean and rms flow velocity}

Figure 6 shows a typical PIV velocity field in the gap region $(z=-3 \mathrm{~mm}, h=10 \mathrm{~mm}$, $\alpha=15^{\circ}$ and $U_{0}=70 \mathrm{~m} / \mathrm{s}$ ). In Ref. [24], it was checked that the velocity fields scale with inflow velocity $U_{0}$. Therefore the mean relative chord-wise $\left(U / U_{0}\right)$ and normal-to-chord $\left(V / U_{0}\right)$ velocities are plotted on Figure 6(a) and (b) respectively whereas the corresponding relative rms values $u^{\prime} / U_{0}$ and $v^{\prime} / U_{0}$ are plotted on Figure 6(c) and (d) respectively. The coloured spots on the rms plots as well as the white areas along the pressure side are due to light reflections by the airfoil or dust particles on the window. As for the tip edge pressure coefficient, the mean velocity is most perturbed in the mid-chord region where the gap cross-flow is strongest. Indeed, the pressure difference between the two airfoil sides generates a very strong jet-like flow that exits the gap at the suction side in a cross-chord direction near $x / c \sim 0.5$. The velocity of this side jet reaches $\sim 1.5$ times the free stream velocity. As it leaves the gap, this jet is deviated by the external flow and rolls up into the tip leakage vortex which is evidenced on Figure 6(c) and Figure 6(d) by two highly turbulent regions: indeed the $2 \mathrm{D}$ map is similar to the $2 \mathrm{D}$ fields extracted from 3D data in [7] where the tip vortex is clearly shown. A region of low chord-wise velocity starts near the suction side at $x / c=0.5$ and convects downstream in a direction that is almost parallel to the stream-wise direction (indicated by the long side of the 


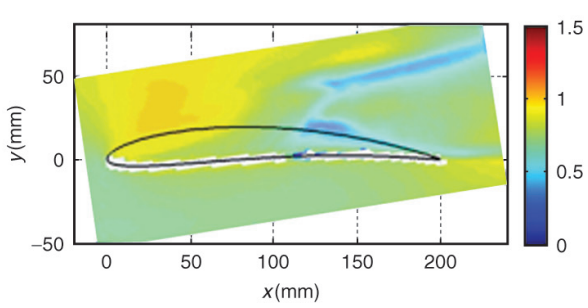

(a) $U / U_{0}$

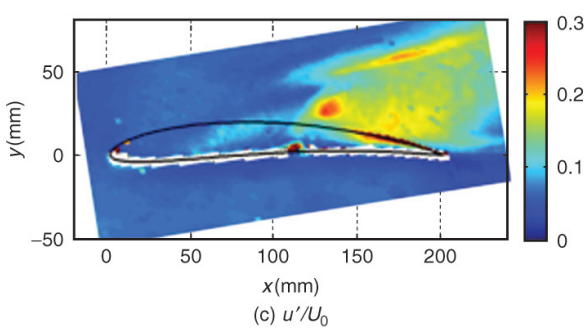

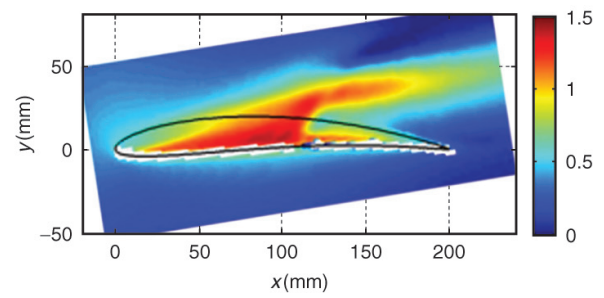

(b) $V / U_{0}$

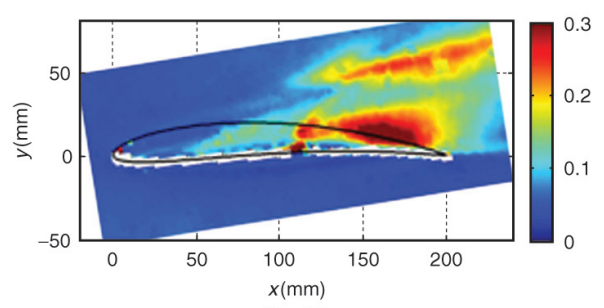

(d) $v^{\prime} / U_{0}$

Figure 6: Velocity field obtained by PIV in the gap (at $z=-3 \mathrm{~mm}$ ) for the reference configuration $\left(U_{0}=70 \mathrm{~m} / \mathrm{s}, h=10 \mathrm{~mm} ; \alpha=15^{\circ}\right)$ : mean chord-wise (a); mean cross-chord (b); rms chord-wise (c); rms cross-chord (d).

rectangular plot). It corresponds to the interaction between the tip clearance flow and the main flow described in refs. [6] and [7]. This region of low chord-wise velocity magnitude $\left(50 \% U_{0}\right)$ and high fluctuation levels evidenced by the outer turbulent shear layer on Figure 6(c) and (d)) is consistent with the tip vortex structure proposed in ref [1]. According to literature, the tangential velocity of the quasi stream-wise tip vortex has a strong component normal to the PIV-plane in this region. Similarly, another region of reduced velocity and high turbulence levels develops nearby the tip/trailing edge corner, much nearer to the airfoil than the other region. In this second region, the flow perturbations are weaker but spread over a larger width into the cross-stream direction. This could correspond to a $2 \mathrm{D}$ cut of the inner side of the tip vortex.

The regions of high turbulence shown on Figure 6(c) and (d) reach their maximum in the downstream third of the airfoil. The region located in the vicinity of the airfoil suction side could be a significant noise source since it almost touches the tip-trailing edge corner which would increase its radiation efficiency. Furthermore, similar measurements [24] carried out at various heights (span-wise cuts) indicate that this inner turbulence region extends into the span-wise direction over the gap height whereas the outer one extends over a quarter chord in the span-wise direction. Moreover, the outer shear layer which is located about 0.2 to 0.4 chord away from the airfoil, is very thin near the bottom plate $(z=-7 \mathrm{~mm}$, not shown here) and becomes broader as $z$ increases. Note that the gap itself is not the most turbulent region. This behaviour, has also been described in cascade flows (e.g. [5],[7]). 


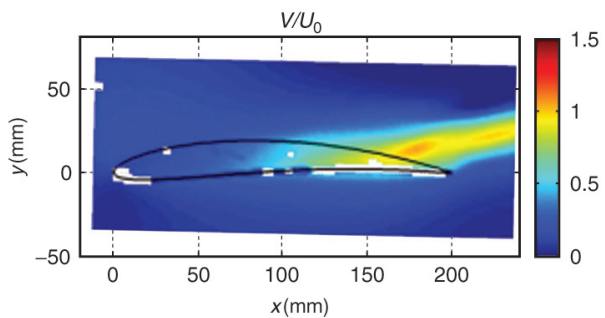

(a) $\alpha=5^{\circ}$

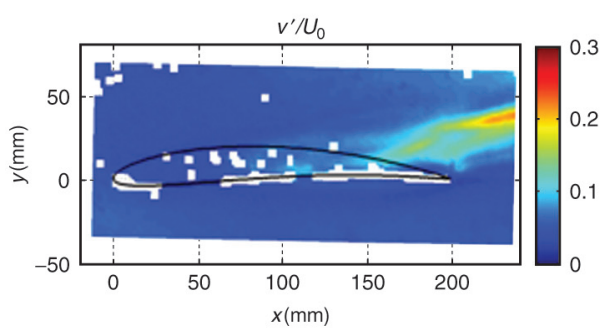

(c) $\alpha=5^{\circ}$

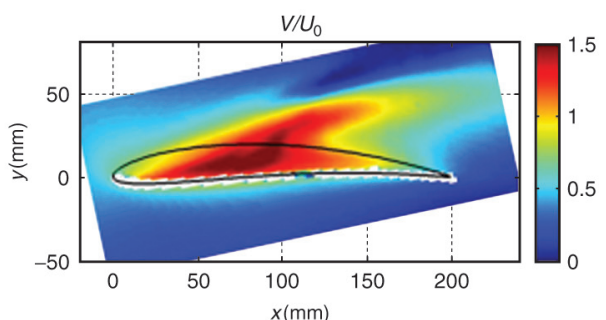

(b) $\alpha=18^{\circ}$

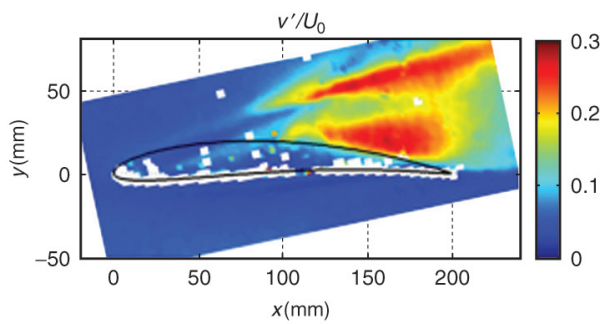

(d) $\alpha=18^{\circ}$

Figure 7: Velocity field obtained by PIV in the gap (at $z=-5 \mathrm{~mm}$ ) for 2 angles of attack $\alpha=5^{\circ}$ (a) (c) and $18^{\circ}$ (b) (d) at $U_{0}=70 \mathrm{~m} / \mathrm{s}$ and $h=10 \mathrm{~mm}$ : mean relative normal-to-chord velocity (a) (b) and relative rms level of normalto-chord velocity fluctuations (c) (d). (white spots are due to light reflections on the airfoil or diffusion or to dust poarticles on the window).

These global features are still found if the flow configuration parameters are changed but their intensity and location varies. The most spectacular changes occur if the angle of attack is modified: as it increases, the gap jet moves upstream: it occurs in the downstream quarter of the airfoil at $\alpha=5^{\circ}$ and almost at the leading edge when $\alpha=18^{\circ}$ as shown on Figure 7. Moreover, the fact that the gap flow scales with the free stream velocity, as already mentioned, means that the physics of this flow are not really velocity dependent in the Reynolds number range considered here $\left(\operatorname{Re}_{c}=0.27-1.2 \times 10^{6}\right)$. As for the gap influence, it was shown by Grilliat et al. [25], that an increase of $h$ also moves the main gap flow region downstream. The trend is quite similar to that shown on Figure 7 and has been reported elsewhere [6],[7].

\section{THE UNSTEADY FLOW}

As it could be expected, the skewed mean cross-flow described in the previous section generates strong unsteady perturbations that are candidate noise sources.

\subsection{Unsteady velocity}

The flow undergoes strong fluctuations in the gap region where eddies of the flat plate boundary layer interact with those generated in the gap. In order to illustrate this 

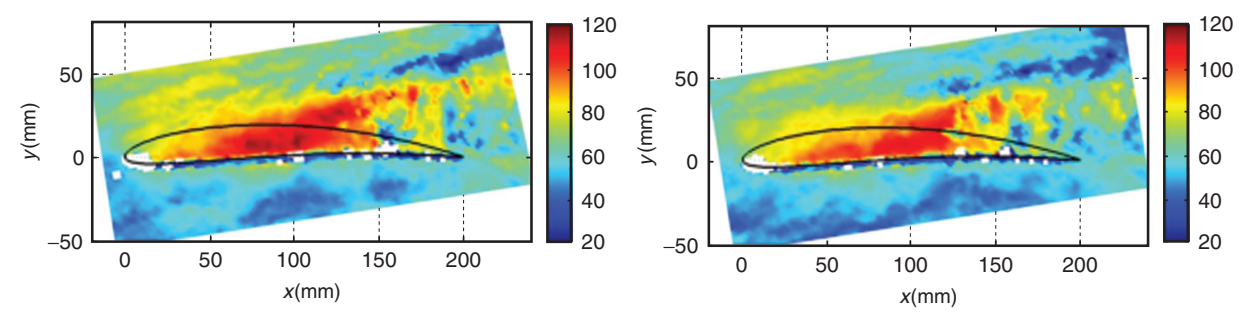

Figure 8: PIV snapshots of the velocity modulus in the gap region for $U_{0}=70 \mathrm{~m} / \mathrm{s}$, $h=10 \mathrm{~mm}$ and $\alpha=15^{\circ}$. The velocity scale is expressed in $\mathrm{m} / \mathrm{s}$. The 2 plots illustrate the diversity of the instantaneous flow patterns. The blue area in top right corner of each plot is a view of the instantaneous detached flow (the blue line on the pressure side as well as white spots are due to Laser reflections on the airfoil and dust particles).

pronounced unsteady behaviour, Figure 8 shows two snapshots of the instantaneous velocity modulus (in fact the modulus of the $2 \mathrm{D}$ velocity in the measurement plane). The accelerated regions can be recognized on each snapshot, although they only slightly resemble to the mean or the rms plots. The snapshots give a good idea about the unsteady nature of the flow and the resulting diversity of instantaneous flow patterns.

Typical chord-wise velocity spectra obtained at $z=-5 \mathrm{~mm}$ inside and outside the gap near the trailing edge $(x / c=0.95)$ are shown on Figure 9 , along with the associated fluctuation levels.

In a region far outside the gap $(y=20-35 \mathrm{~mm})$, a hump around $1.2-1.7 \mathrm{kHz}$ whose limits are not well defined arises clearly from the background level. Moreover, for these positions the broadband spectra are also about $10 \mathrm{~dB}$ higher than for the other positions located inside or nearby the gap $(y=-15$ to $10 \mathrm{~mm})$, except at the low frequency end. The hump as well as the high turbulence level are associated to the outer shear layer described in the previous section (Figure 6). The outer shear layer can also be recognized in the snapshots of Figure 8 . At this advanced chord-wise position $(x / c=$ $0.95)$ the PIV snaphshots from Figure 8 as well as the $v^{\prime}$ rms plots from the previous section also indicate that the gap itself is not anymore a region of intense perturbations. This is confirmed by the present spectra $(y=-15$ to $10 \mathrm{~mm})$ where not only the broadband and overall levels are lower but the hump is also missing.

\subsection{Wall pressure fluctuations}

On Figure 10, typical spectra from the suction side tip edge are traced on plot (a). Plot (b) is obtained by probes located in and nearby the tip clearance at $x / c=0.775$. A hump between 0.7 and $3 \mathrm{kHz}$ typically can be recognized on most spectra, even at the pressure side tip edge (probe 46). Similar features are also found on velocity spectra in this region and the hump corresponds to that found in the outer shear layer on Figure 9. This confirms the scenario of the roll-up mechanism: perturbations generated in the gap at $3 / 4$ chord are expelled from the gap and convected to the outer shear layer of the tip 


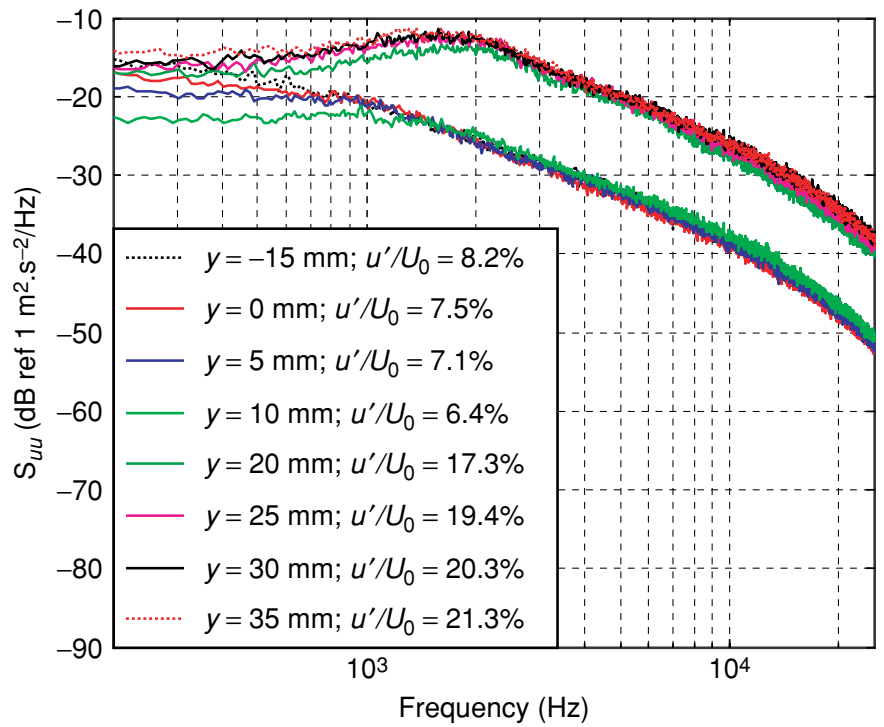

Figure 9: $\quad$ Spectra in the gap region at $x / c=0.95 z=-5 \mathrm{~mm}$ and $y=-15 ; 0 ; 5 ; 10$; $20 ; 25 ; 30 ; 35 \mathrm{~mm}: u^{\prime} / U_{0}\left(u^{\prime}=5.76 ; 5.24 ; 4.97 ; 4.48 ; 12.1 ; 13.6 ; 14.2\right.$; $14.9 \mathrm{~m} / \mathrm{s}$ respectively) $\left(U_{0}=70 \mathrm{~m} / \mathrm{s}, h=10 \mathrm{~mm} ; \alpha=15^{\circ}\right)$.

(a)

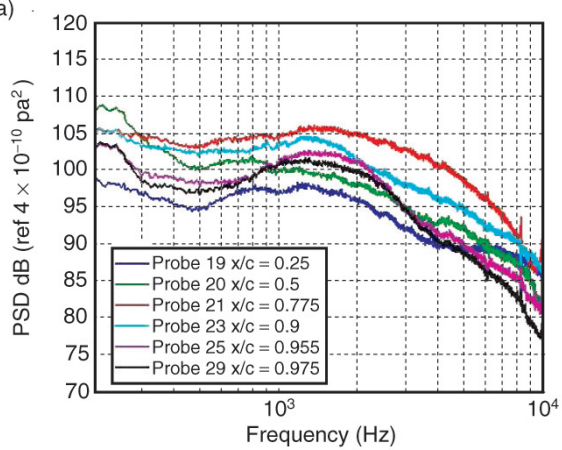

(c)

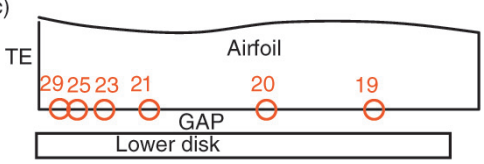

(b)

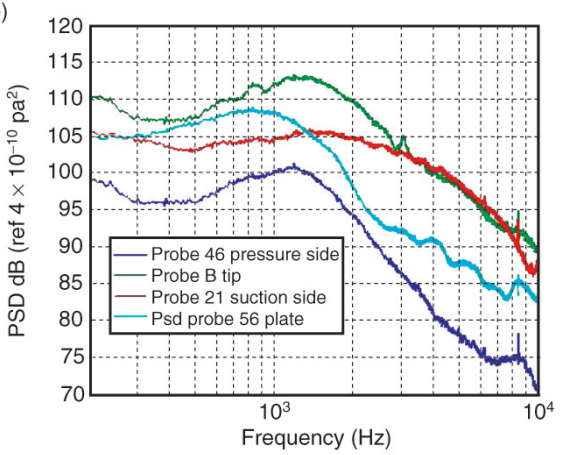

(d)

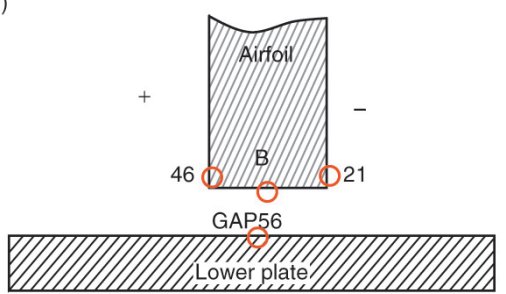

Figure 10: Wall pressure spectra in the tip region: (a) along the tip suction side at various chord-wise positions sketched in (c); (b) in the gap at $x / c=0.775$ at various points sketched in (d) - $\left(h=10 \mathrm{~mm}, \alpha=15^{\circ}, U_{0}=70 \mathrm{~m} / \mathrm{s}\right)$. 
vortex. The relatively high broadband level of the velocity spectra which is not obvious in the gap pressure spectra, is probably due to turbulence from the casing wall boundary layer entrained by the tip vortex. The maximum of the wall pressure hump lies about $1.4 \mathrm{kHz}$, which corresponds to a chord based Strouhal number $\mathrm{St}_{c}$ about 4 . It is however not so clearly recognizable upstream of the tip jet (probe 19) described in section 3 and it extends to very high frequencies for probe 21 which is located just above the upper shear layer of the tip jet where it leaves the gap. This shear layer can possibly be accounted for additional higher frequency perturbations: the frequency range comprised between 4 and $7 \mathrm{kHz}$ corresponds to a gap based Strouhal number $\mathrm{St}_{h}=f h / V_{\max }$ (where $V_{\max } \sim 100 \mathrm{~m} / \mathrm{s}$ is the tip jet velocity) that varies between 0.4 and 0.7 which is typical for subsonic jets. In [25], similar results are reported for small gaps down to $h=3 \mathrm{~mm}(h / c$ $\sim 0.15$ ). For $h \leq 3 \mathrm{~mm}$ the hump disappears and the pressure perturbations become very small inside the gap, whereas for $h \geq 10 \mathrm{~mm}$, the perturbations become independent from the gap which means that the influence of the casing wall vanishes.

\section{RADIATED SOUND}

\subsection{The far field}

The far field measurements show that the noise generated by the tip clearance flow is of the same order of magnitude as the distributed trailing edge noise for particular frequency ranges and that it even dominates at specific frequency ranges depending on the observer position. In order to illustrate this, the difference between the sound measured with and without an $h=10 \mathrm{~mm}$ gap is plotted on Figure 11. The PSD level is computed for frequencies at which the $h=10 \mathrm{~mm}$ configuration is louder than the no gap configuration. This allows to identifying the far field contribution of the tip clearance, the background noise and the distributed trailing edge noise being removed. The result is plotted for the $h=10 \mathrm{~mm}, \alpha=15^{\circ}, U_{0}=70 \mathrm{~m} / \mathrm{s}$ configuration as a frequency/observer angle diagram on Figure 11.

The first noise component is pointed at by the pink ellipses on the figure: it is found that the PSD level varies according to the $5^{\text {th }}$ power of the inflow velocity and can be related to the interaction between turbulent structures generated in the gap and the airfoil suction side edge. Indeed the frequency range corresponds to the medium frequency hump found in the pressure spectra that are already felt inside the gap (see Figure 10(b)). These structures are generated by the flow separation on the gap pressure side edge but radiate after they leave the gap region, probably as they are swept past the trailing edge, which explains the power law and the observed directivity. As shown in ref [20] this source was qualitatively modelled with a modified trailing edge noise model based on an extension to oblique 3D gusts and out of mid-span plane [26][27] of Amiet's theory [28]. For the tip/trailing edge noise model, the source concentration in the tip region was taken into account via a span-wise damping coefficient that could be fitted experimentally from the span-wise decay of the wall pressure spectra, whereas the bottom plate was simply replaced by an image airfoil.

The second source, ranging higher in the frequency domain, typically between 4 and $7 \mathrm{kHz}$, is found to vary according to a power of the inflow velocity comprised between 7 and 8. It is located on the upstream part of the jet-like flow that leaves the gap on the 


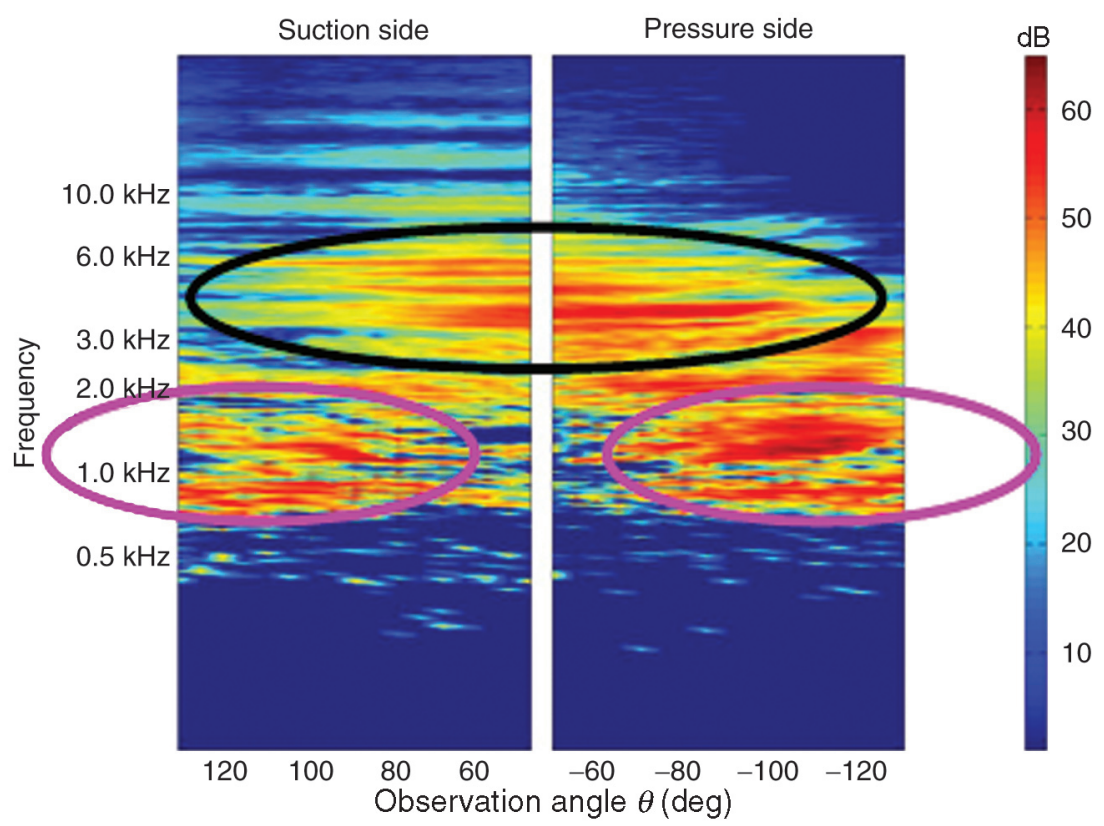

Figure 11: Tip clearance contribution to the far field ( $h=10 \mathrm{~mm}, \alpha=15^{\circ}, U_{0}=70 \mathrm{~m} / \mathrm{s}$ ), against observation angle and frequency. The color scale indicates the PSD level in dB-ref $4.10^{-10} \mathrm{~Pa}^{2} / \mathrm{Hz}$.

airfoil suction side and is therefore related to secondary turbulent eddies generated by the flow separation at the gap suction side edge: the jet-like structure of the flow expelled in the upper tip flow thus explains the velocity dependence of this source.

Both sources are of the same order of magnitude as the resultant self noise of the whole trailing edge in their respective frequency domain. Thus the tip source is both an important source and a source that is difficult to measure. In turbomachinery applications however, it should be outranged by the interaction of the fan tip wakes with the OGV blades. Moreover, due to the very small tip clearance achieved in modern UHBR fans, the leakage flow is likely to be replaced by a different vortical flow around the blade or simply to be outranged by the trailing edge self noise which will be relatively more powerful due the large span.

\subsection{The near and far unsteady field}

On Figure 13 the coherence is plotted between the far field microphone located $1 \mathrm{~m}$ across the suction side and the LDA probe located at various points along the line shown on Figure 12.

Results indicate that for frequencies comprised between 0.5 and $1.5 \mathrm{kHz}$ typically, the far field is coherent with the velocity field in the vicinity of the wall ( $\eta<6$ to $7 \mathrm{~mm}$ ) the maximal coherence being obtained at $\eta=3-5 \mathrm{~mm}$ and $f \sim 1 \mathrm{kHz}$ on the $U$ component. 

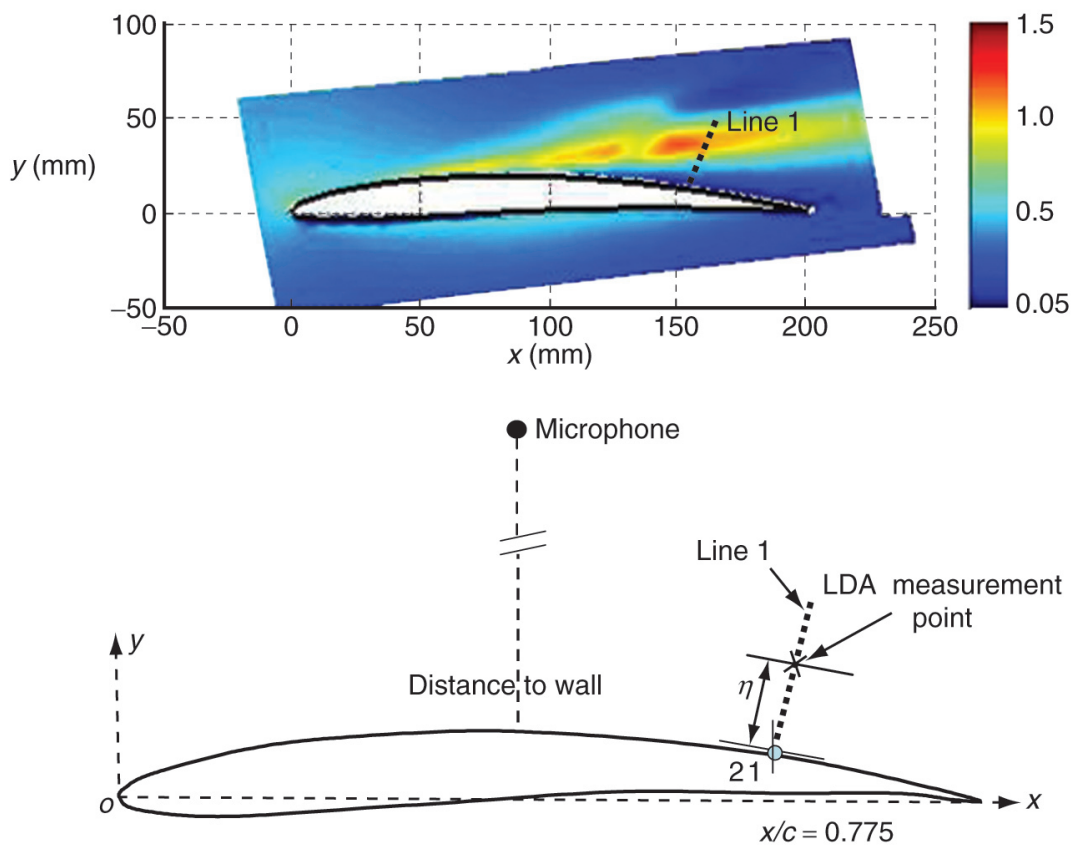

Figure 12: Sketch of joint LDA-pressure measurements. Top: V component of velocity (normal-to chord) at $z=1 \mathrm{~mm}$ and LDA measurement line (Line 1) for LDA-far field pressure coherence measurements. Bottom: same without velocity field but with extra details: $\eta$ is the distance from the current measurement point to the wall at the position of probe 21 $(x=0.775 \mathrm{c}=155 \mathrm{~mm}$ on the suction side). The microphone is $1 \mathrm{~m}$ across the suction side at $x / c=0.5$. Velocity is non-dimensioned by the upstream velocity $U_{0}$. $\left(U_{0}=70 \mathrm{~m} / \mathrm{s}, h=10 \mathrm{~mm} ; \alpha=15^{\circ}\right)$.

It should be mentioned that although the values of the coherence remain quite low (less than 0.2), they are actually remarkably high, given the fact that each LDA measurement volume is indeed very small and that it is expected to contribute only weakly to the far field. This means that although the source is due to turbulence, it is quite concentrated in a narrow region near the airfoil tail. In other words, the sources of sound in the medium frequency range are due to eddies passing nearby the trailing edge corner. This is consistent with the low frequency source $5^{\text {th }}$ power velocity dependence mentioned in the previous subsection.

Similar conclusions have been drawn by Grilliat et al. [25] from Hot Wire Anemometry - wall pressure coherence measurements. Grilliat et al. also examined the shear layers in the flow region where the tip jet exits from the gap and found evidence of the high frequency jet-like noise contribution. 

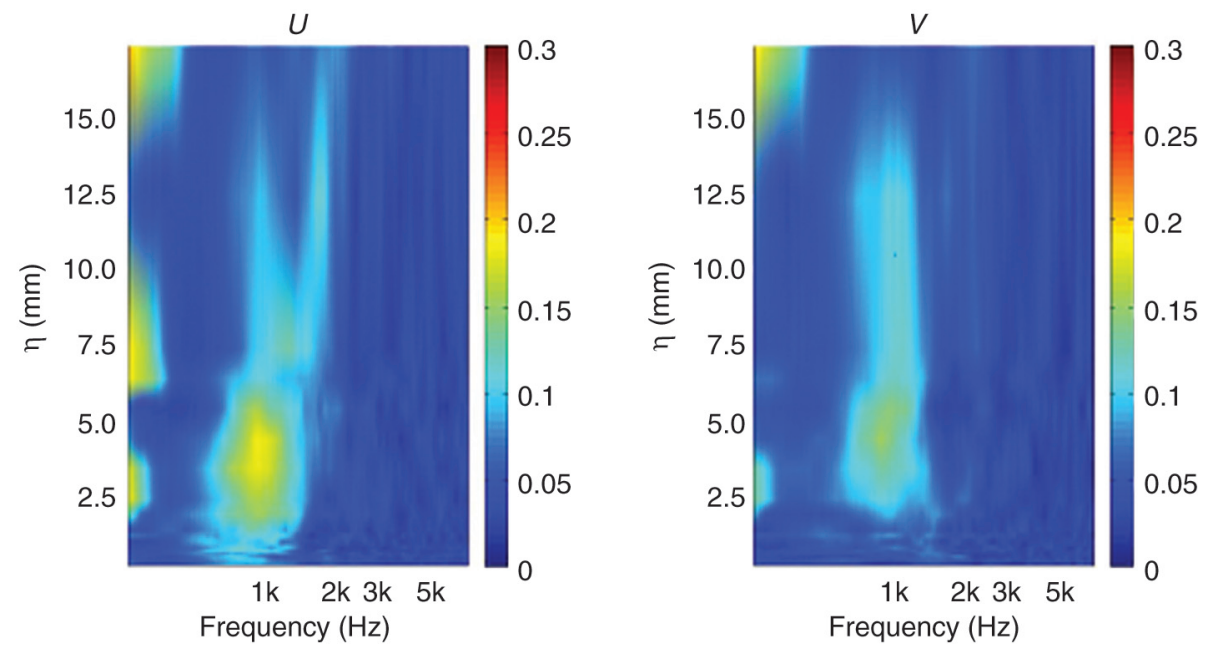

Figure 13: Coherence between the velocity measured by LDA in the plane of probe $21(z=1.5 \mathrm{~mm})$ and the far field microphone for various LDA probe positions following line 1. $\eta$ denotes the distance between the LDA measurement point and the wall along the measurement line.

\section{CONCLUSIONS}

Due to their 3D nature, the sound generation mechanisms of the tip clearance self induced perturbations are very difficult to model. The present experimental study is a first step towards a better understanding of tip clearance flows and the associated sound production.

A strong tip flow was obtained in this non-rotating, low Mach number single airfoil configuration by setting a high angle of attack to an already cambered airfoil. The strong gap flow was found to be mainly concentrated in a quite narrow part of the airfoil (less than half the chord) whose location varies with the angle of attack and the gap. This high speed flow exits the gap as a cross-flow jet that is deflected by the surrounding flow and rolls up into a large tip vortex. Since the tip vortex interacts with the outer flow and the airfoil wall, it yields two highly turbulent shear layers, one located in the outer flow and one near the wall. As a result, this aerodynamic study allowed identifying major features of the flow that are candidate sources mechanisms:

- the jet-like clearance flow is believed to generate sound when leaving the clearance region either directly or by interacting with the blade tip edge(s);

- the TLV feeds unsteady perturbations into the blade wall pressure field that become sound sources as they are scattered by the tip edge and/or the trailing edge corner. 
Although one could add possible interaction noise sources due to ingestion of incoming disturbances by the tip clearance flow, these were not found to be significant in the present study which was carried out with a quiet inflow.

The far field and joint acoustic/aerodynamic measurements confirmed this analysis.

As for the application to fans, the large gaps studied here find of course a natural application in low speed applications. However, the mechanisms involved in the sound generation are likely to be relevant for turbomachinery applications as far as some specifics are taken into account: the major difference between a fan and the present experiment is that there is no relative motion between the casing and the blade in the configuration addressed here. Moreover, the rotation in a real turbo engine fan probably results in a larger distance between the blade and the tip vortex, which would be a real issue in terms of sound production. Indeed, the vicinity of the tip vortex and the blade trailing edge plays a decisive role in the sound production. Furthermore, as mentioned in the introduction, rotating instabilities take place upstream of the impeller. These points need to be properly addressed by detailed investigations of the tip flows in real UHBR fans since the adjacent blade may set a limit to the tip vortex spreading. However, the goal of the present study was to avoid superimposing too many flow phenomena in order to identify and characterize a few particular mechanisms of tip clearance noise.

\section{ACKNOWLEDGEMENTS}

This work has been funded by the European Community as part of the $6^{\text {th }}$ Framework Project PROBAND no AST4-CT-2005-012222.

\section{REFERENCES}

[1] Bindon, J.P., The measurement \& Formation of Tip Clearance Loss, Journal of turbomachinery, 111, 257-263, (1989).

[2] Inoue, M. And Kuroumaru, M., Structure of Tip Clearance Flow in an Isolated Axial Compressor Rotor, Transactions of the ASME, 111, 250-256, (1989).

[3] Storer, J.A. and Cumpsty, N.A., Tip Leakage Flow in Axial Compressors, Transactions of the ASME, 113, 252-259, (1991).

[4] Lakshlinarayana, B., Zaccaria, M. and Marathe, B., The Structure of Tip Clearance Flow in Axial Flow Compressors, Transactions of the ASME, 117, 336-347, (1995).

[5] Muthanna C., Flowfield Downstream of a Compressor Cascade with Tip Leakage, Ms Thesis, Faculty of the Virginia Polytechnic Institute and State University, (1998).

[6] TAng, G., Measurements of the Tip-gap Turbulent Flow Structure in a Low-speed Compressor Cascade, Ph.d. thesis, Faculty of the Virginia Polytechnic Institute and State University, (2004).

[7] Intarater, N., Formation and Development of the Tip Leakage Vortex in a 
Simulated Axial Compressor with Unsteady Inflow, Ph.d. thesis, Faculty of the Virginia Polytechnic Institute and State University, (2006).

[8] You, D., Wang, M., Moin, P. and Mittal, M., Effects of tip-leakage flow in a turbomachinery cascade, Physics of Fluids, 18(10), (2006).

[9] Fukano, T. And TAKamatsu, Y., The effects of tip clearance on the noise of lowpressure axial and mixed flow fans, Journal of Sound and Vibration, 105, 291-308 (1986).

[10] Fukano, T. And JAng, C., Tip clearance noise of axial flow fans operating at design and off-design condition, Journal of Sound and Vibration, 275, 1027-1050 (2004).

[11] Ganz U.W., Patten T.J., Scharpf D.F. and Joppa P.D.: Boeing 18-inch fan rig broadband noise test. NASA CR-1998-208704, (1998).

[12] Kameier, F., Neise, W., Rotating blade flow instability as a source of noise in axial turbomachines, Journal of Sound and Vibration, 203(5), 833-853, (1997).

[13] Kameier, F., Neise, W., Experimental study of tip clearance losses and noise in axial turbomachines and their reduction, Journal of Turbomachinery, ASME Trans., 119, 460-471, (1997).

[14] Bae, J.W., Breuer, K.S. AND Tan, C.S., Active control on tip flow in axial compressors, Journal of Turbomachinery, 127, 352-362, (2005).

[15] Khourrami, M.R. And Choudari, M., A novel approach for reducing rotor tipclearance induced noise in turbofan engines, AIAA-paper no 2001-2148, (2001).

[16] Neuhaus, L., Neise, W., Active flow control to improve the aerodynamic and acoustic performance of axial turbomachines, AIAA paper no 2002-2948, in Proceedings of the 1rst Flow Control Conference, St. Louis, MO, USA, June 24-26, (2002).

[17] Corsini, A., Perugini, B., Rispoli, F., Sheard, A.G. And Kinghorn, I.R., Experimental and numerical investigation on passive devices for tip clearance induced noise reduction in axial flow fans, $7^{\text {th }}$ European Conference on Turbomachinery (ECT-7), 5-9 March Athens, Greece 2005.

[18] JACOB, M.C., Boudet, J., CASAlino, D., Michard, M., A od-airfoil experiment as benchmark for broadband noise modelling, Theoretical and Computational Fluid Dynamics, 19(3), 171-196, (2005).

[19] Camussi, R., Caputi Gennaro, G., Jacob, M. C., Grilliat, J., Experimental study of a tip leakage flow - part two: wavelet analysis of wall pressure fluctuations, AIAA paper no 2007-3685, Proceedings of the $13^{\text {th }} A I A A / C E A S$ Aeroacoustics Conference, Rome, Italy, May 21-23, (2007).

[20] Grilliat, J., Jondeau, E., Roger, M., Jacob, M. C., Camussi, R., Broadband noise prediction models and measurements of tip leakage flows, AIAA paper no 2008-2845, Proceedings of the $14^{\text {th }}$ AIAA/CEAS Aeroacoustics Conference, Vancouver, BC, May 5-7, (2008).

[21] CAmussi, R., Robert, G., JACOB, M. C., Cross-Wavelet analysis of wall pressure fluctuations beneath incompressible turbulent boundary layers, Journal of Fluid Mechanics 167, 11-30, (2008). 
[22] Roger, M. And Perennes, S., Aerodynamic noise of a two-dimensional wing with high-lift devices, AIAA-paper no 98-2338, (1998).

[23] Arguillat, B., Etude expérimentale et numérique de champs de pression pariétale dans l'espace des nombres d'onde, avec application aux vitrages automobiles, Thèse de Docteur en Mécanique des Fluides et Acoustique, No d'ordre 2006-14, Ecole Centrale de Lyon, France, (2006).

[24] Jacob, M. C., Camussi, R., Caputi Genaro, G., Grilliat, J., Jondeau, E., RozenBerg, Y., Deliverable D2.5: T2.1 Experiment, Final Report: PROBAND, AST4-CT-2005-012222, March, (2007).

[25] Grilliat, J., Jacob, M. C., Camussi, R., Caputi Gennaro, G., Experimental study of a tip leakage flow - part one: aerodynamic and acoustic measurements, AIAA paper no 2007-3684, Proceedings of the 13 $3^{\text {th }}$ AIAA/CEAS Aeroacoustics Conference, Rome, Italy, may 21-23, (2007).

[26] Roger, M., Moreau, S., Back-scattering correction and further extensions of Amiet's trailing-edge noise model. part 1: Theory, Journal of Sound and Vibration, 286, p 477-506, (2005)

[27] Moreau, S., Roger, M., Back-scattering correction and further extensions of Amiet's trailing-edge noise model. Part II: Application Journal of Sound and Vibration, 323, 397-425, (2009).

[28] Амiet, R. K., Noise due to turbulent flow past a trailing edge, Journal of Sound and Vibration, 47(4), 387-393, (1976). 